\title{
Single-Phase Natural Circulation in a PWR during a Loss of Coolant Accident
}

\author{
Mohammed W. Abdulrahman', Mikdam M. Saleh², Jonathan Anand \\ ${ }^{1}$ Rochester Institute of Technology \\ Dubai, UAE \\ mwacad@rit.edu; mikdam2@yahoo.com \\ ${ }^{2}$ University of Baghdad \\ Baghdad, Iraq \\ ja3127@rit.edu
}

\begin{abstract}
In all light water reactors (LWRs), natural circulation is an important passive heat removal mechanism. To explain the effects of diminished primary coolant inventory on natural circulation, an analytical model is derived. The analysis is based on a one-dimensional model and the quasi-steady hypothesis in which the continuity, momentum, and energy equations are solved. Expressions for mass flow rate, and temperature distributions are derived, and the effect of the core power is investigated. The model covers the mode of a singlephase natural circulation. Comparisons with the experimental results of a previous work are presented and show reasonable agreement with the analytical results.
\end{abstract}

Keywords: natural circulation; PWR; single-phase; LOCA

\section{Introduction}

Since the Three Mile Island accident, natural circulation (NC) has become an increasingly important light water reactor (LWR) safety issue. It represents a unique and passive means of cooling during certain kinds of accidents or transients in a pressurized water reactor (PWR) such as small break loss-of-coolant accidents (LOCAs) or operational transients involving loss of pumped circulation. The only requirement for the $\mathrm{NC}$ occurrence is a hydrostatic head differential between the heat source (core) and the heat sink (steam generator), so that the cooled fluid and condensed steam, if any, can recirculate.

For PWRs, there are four identified modes of $\mathrm{NC}$ as quality increases:

- Single-phase water flow, in which the water is sub-cooled and there are no voids;

- Combined single and two-phase flow, in which both sub-cooled water and voids are formed;

- Two-phase flow, in which voids formed by core heat addition are circulated and condensed;

- Reflux condensation, where single-phase steam flow is condensed in the steam generator.

The important parameter governing heat removal for the first three modes is the loop flow rate. There are many studies that have been performed to investigate NCs. Ybarrondo et. Al, reviewed the calculation procedures used for predicting the thermal and hydraulic response of commercial power reactors to a loss-of-coolant accident (LOCA). The computational models required for each phase of the accident were reviewed [1]. Lewis, presented a simplified treatment of a single-phase loop of a nuclear reactor which yields an estimate of the steady-state flow rate and core temperature difference. He employed an overall heat balance on the core rather than the formal energy equation. Instead of the density integral in the momentum equation, an equivalent "driving head" was introduced, with two distinct values of the densities (for the hotter and colder portions of the loop) [2]. Zvirin et al., carried out a theoretical and experimental study of single-phase NC in an apparatus with parallel loops. Their system, was relevant to a PWR. Tests were performed for steady-state and transient conditions with heat removal from either or both heat exchangers. The analysis utilized the assumption of single equivalent loop and was based on existing one-dimensional modelling methods for thermosyphons. The coupled momentum and energy equations for the fluid in the loop were solved to yield the steady-state flow rate, temperature distributions and the transient behaviour of the loop. It was shown that the core flow resistance, input heat distribution, and upper plenum geometry yielded three-dimensional flow effects, which contributed to the overall difference of $30 \%$ between the analytical and experimental results [3]. Abdulrahman has studied the analytical model of the 2-dimensional steady-state heat transfer equation through a packed bed reactor and calculated the temperature distributions inside the reactor $[4,5]$. 
The objective of this work is to develop a theoretical model to analyse the single-phase NC phenomena relevant to small breaks for PWRs. A fundamental tenet of this work is that the entire circulation process can be treated as quasisteady. The model utilizes the one-dimensional approach and the quasi-steady hypothesis and solves analytically the loop momentum balance together with the conservation of mass and energy, and develops expressions for the core flow rate and core inlet and outlet temperature as a function of primary pressure. The model should be capable of analysing the single-phase mode of NC. In addition, the model should be able to forgo the usual assumptions of linear temperature distribution along the steam generator $[3,6]$ by actually incorporating the overall heat transfer coefficient in the theoretical analysis.

\section{Steady State One-Dimensional Natural Circulation Flow}

To describe NC in a PWR loop, it is preferred to follow the conventional formulations utilizing mass, momentum and energy conservations. Before proceeding with the mathematical development, the following assumptions are considered:

1. Only one spatial coordinate, $s$, which runs around the loop is considered (Fig. 1).

2. The average cross-sectional temperature $T$, is equal to the mixed mean (or bulk) temperature.

3. In the free convection loop, the pressure changes are negligible as compared to system pressure so that the fluid is considered incompressible.

4. The cross-sectional area of the flow is taken as a constant that is equal to the average cross-sectional area of the flow.

5. Fluid properties, except the density, are considered constants.

6. Only the up-flow side of steam generator will be regarded as effective in removing heat and consequently the heat removal from the down-flow side of steam generator will be neglected. This is due to the fact that the primary temperature will approximately equilibrate with that of the secondary in the up-flow side of steam generator.

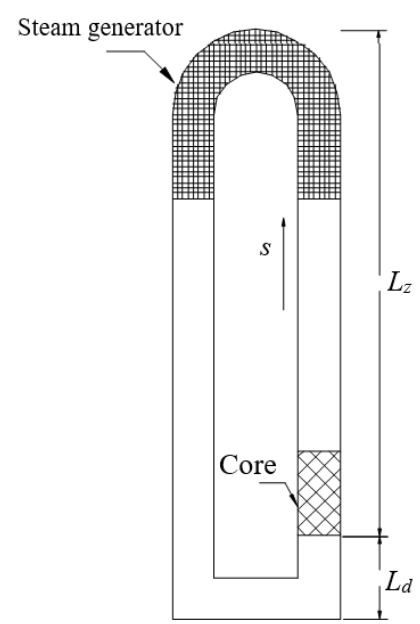

Fig. 1. One-dimensional loop.

For a flow in a one-dimensional positive $s$-direction, the steady state equations of continuity, momentum, and energy are respectively [7];

$$
\begin{gathered}
\frac{d u}{d s}=-\frac{u}{\rho} \frac{d \rho}{d s} \\
\rho u \frac{d u}{d s}=-\frac{d P}{d s}-F_{s}-\rho g \cos (\theta)
\end{gathered}
$$




$$
\rho A u \frac{d h}{d s}=-\frac{d q}{d s}
$$

where $u$ is the flow velocity, $\rho$ is the fluid density, $P$ is the primary pressure, $g$ is the gravitational acceleration, $A$ is the is the cross sectional area of the flow channel, $h$ is the specific enthalpy, and $q$ is the heat added to (or rejected from) the coolant. In terms of the mass flow rate $W$, the flow velocity $u$ is:

$$
u=\frac{W}{\rho A}
$$

For turbulent flow in a circular tube of diameter $D$, the viscous force per unit volume $F_{S}$ is;

$$
F_{S}=\frac{4 f\left(\frac{1}{2} \rho u^{2}\right)}{D}
$$

where the friction factor $f$ is given by $[8] ; f=\frac{0.046}{(R e)^{0.2}}$

Substituting Eqs. (1), (4) and (5) into Eq. (2) and integrate around the loop, the momentum Eq. will be;

$$
\oint \frac{W^{2}}{A^{2}(s)} \frac{d v}{d s} d s=-\oint \frac{d P}{d s} d s-\oint \frac{4\left(\frac{1}{2} f W^{2}\right)}{\rho A^{2}(s) D(s)} d s-\oint \rho g \cos (\theta) d s
$$

where $v$ is the fluid specific volume and $P$ is the primary pressure. The left-hand side and the pressure term of Eq. (6) will vanish. For strongly turbulent flow, it can be assumed that the friction coefficient is constant for a given primary pressure. Noting that $A$ and $D$ are given by their average values over the loop and that $d z=d s \cos (\theta)$, then Eq. (6) becomes;

$$
-g \oint \rho d z=\frac{\frac{1}{2}(4 \bar{f}) W^{2}}{\bar{A}^{2} \bar{D}} \oint \frac{d s}{\rho}
$$

From Fig. 1;

$$
\oint \rho d z=\int_{0}^{L_{z}} \rho_{1} d z+\int_{L_{z}}^{0} \rho_{1} d z+\int_{0}^{-L_{d}} \rho_{2} d z+\int_{-L_{d}}^{0} \rho_{2} d z=\int_{0}^{L_{z}} \rho_{1} d z-\int_{0}^{L_{z}} \rho_{2} d z=\left(\bar{\rho}_{1}-\bar{\rho}_{2}\right) L_{z}
$$

and;

$$
\oint \frac{d s}{\rho}=\oint v d s=\bar{v} L_{t}
$$

where $\rho_{1}$ and $\rho_{2}$ are the fluid densities of the system up-flow and down-flow sides respectively, $\bar{\rho}_{1}$ and $\bar{\rho}_{2}$ are the average values of the fluid densities $\rho_{1}$ and $\rho_{2}$ respectively, $\bar{v}$ is the average value of the fluid specific volume, $L_{z}$, and $L_{d}$ are the heights of the PWR and downcomer systems respectively, and $L_{t}$ is the total circulation length. Substituting Eqs. (8) and (9) into Eq. (7) and rearranging, to get; 


$$
\frac{\frac{1}{2}(4 \bar{f}) W^{2}}{\bar{A}^{2} \bar{D}} \bar{v} L_{t}=g\left(\bar{\rho}_{2}-\bar{\rho}_{1}\right) L_{z}
$$

Define:

$$
\widehat{W}^{2}=\frac{2 g L_{z} \bar{D} \bar{\rho}_{o}^{2} \bar{A}^{2}}{4 \bar{f}_{o} L_{t}}
$$

where $\bar{D}$ and $\bar{A}$ are the average diameter and cross sectional area of the flow channel respectively. Note that $\widehat{W}$ has the dimensions of flow rate and $\bar{f}_{o}$ and $\bar{\rho}_{o}$ are respectively the overall system average friction coefficient and density corresponding to the conditions at the beginning of NC. Substituting Eq. (11) in Eq. (10) and solving for the mass flow rate, to get;

$$
W=\frac{\widehat{W}}{\bar{\rho}_{o}} \sqrt{\frac{\left(\bar{\rho}_{2}-\bar{\rho}_{1)}\right.}{\bar{v}} \frac{\bar{f}_{o}}{\bar{f}}}
$$

where $\bar{f}$ is the average friction factor. The energy Eq. (10) can be written separately for each component of the loop. The term $\left(-\frac{d q}{d s}\right)$ in Eq. (10) is equal to $\left(-\bar{U} \pi D_{s} n\left(T-T_{\text {sec }}\right)\right)$ for the heat sink and zero for the (insulated) pipes. For the heated section, it depends upon the input power distribution. For incompressible single-phase flow, it will be assumed that the Boussinesq approximations for density are valid. Thus; $\rho=\rho_{l}\left[1-\beta\left(T-T_{l}\right)\right]$. This equation might be regarded as a Taylormode equation of state and can be written as;

$$
\rho=a+b T
$$

where $T$ is the fluid temperature, and the constants $a$ and $b$ can be calculated from:

$a=\rho_{l}\left(1+\beta T_{l}\right)$ and $b=-\rho_{l} \beta$

where $\rho_{l}$ is the density reference value that is evaluated at some reference temperature $T_{l}$ and $\beta$ is the volume expansion coefficient that is defined by:

$$
\beta=\frac{1}{v}\left(\frac{\partial v}{\partial T}\right)_{P}
$$

It is well known that the power distribution in a typical PWR core is far from being uniform. To first approximation, the distribution may be taken as sinusoidal. In this paper, to simplify derivations, uniform input power distribution is taken into consideration. For a uniformly distributed input power, the term $\left(-\frac{d q}{d s}\right)$ is equal to $\frac{Q_{o}}{L_{c}}$ for the heated section. Hence, Eq. (10) can be written as: 


$$
W \frac{d h}{d s}=\left\{\begin{array}{cc}
Q_{o} / L_{c} & \text { (heat source) } \\
-\bar{U} \pi D_{s} n\left(T_{s}-T_{\text {sec }}\right) & \text { (heat sink) } \\
0 & \text { (insulated pipes) }
\end{array}\right.
$$

where $Q_{o}$ is the heat input power, $L_{c}$ is the length of the core, $\bar{U}$ is the average value of the overall heat transfer coefficient, $D_{s}$ is the steam generator diameter, $n$ is the number of primary tubes of steam generator, $T_{s}$ and $T_{\text {sec }}$ are are the steam generator temperatures of the primary and secondary sides respectively.

\subsection{Single-Phase Loop Flow}

Consider the loop to contain liquid only, as shown in Fig. 2. For single-phase, the fluid enthalpy may be taken as, $d h=$ $C d T$, and hence Eqs. (15) yield;

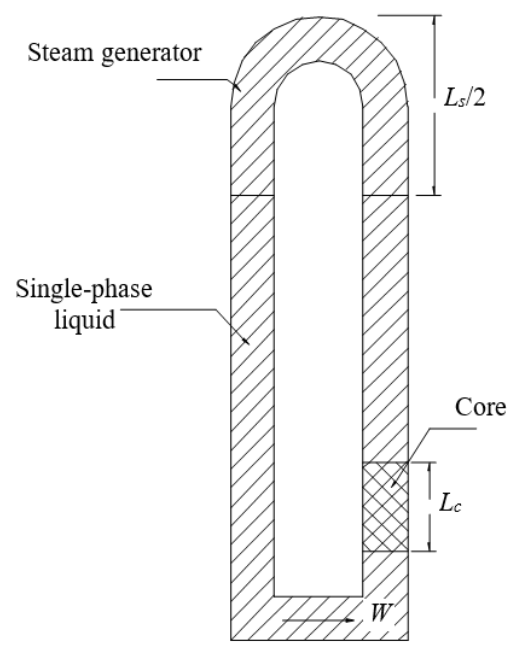

Fig. 2. Single-phase loop.

$$
W C \frac{d T}{d s}=\left\{\begin{array}{cc}
Q_{o} / L_{c} & \text { (heat source) } \\
-\bar{U} \pi D_{s} n\left(T_{s}-T_{s e c}\right) & \text { (heat sink) } \\
0 & \text { (insulated pipes) }
\end{array}\right.
$$

where $C$ is the specific heat capacity that is defined by; $C=\frac{h_{o}-h_{i}}{T_{o}-T_{i}}$. The solution of Eq. (16c) yields a uniform temperature distribution in the connecting pipes, which requires, then, that the core outlet and the steam generator inlet temperatures to be at the same temperature. To get the temperature distribution of the core, $T_{c}$, Eq. (16a) must be solved. The exact solution of Eq. (16a) for a uniformly distributed input power gives a linear temperature profile in the core, thus; noting that $s=z$; then,

$$
T_{C}=T_{i}+\frac{Q_{o}}{C W} \frac{z}{L_{c}} \quad\left(0 \leq z \leq L_{c}\right)
$$

where $T_{i}$ is the core inlet temperature, and $z$, is the distance measured from the bottom of the heated section. The outlet temperature of the core, $T_{o}$, is obtained from Eq. (17) by setting $z=L_{c}$, thus; 


$$
T_{o}=T_{i}+\frac{Q_{o}}{C W}
$$

The relation (18) yields the temperature difference of the core $\left(\Delta T_{\text {core }}\right)$ (or between the hot and cold legs).

$$
\Delta T_{\text {core }}=T_{o}-T_{i}=\frac{Q_{o}}{C W}
$$

The most important parameter which can serve to characterize the system behaviour at steady state is the temperature difference over the heated section, which is directly related to the flow rate in the loop, Eq. (19). To get the temperature distribution of the primary side of the steam generator, Eq. (16b), can be readily integrated to give:

$$
T_{S}=T_{s e c}+\left(T_{o}-T_{s e c}\right) e^{-\frac{\bar{U}_{1} \pi D_{s} n}{C W} z} \quad\left(0 \leq z \leq L_{S}\right)
$$

where $\bar{U}_{1}$ is the average overall single-phase heat transfer coefficient, and $z$, is measured from the bottom of the primary side of the hot leg of the steam generator. The inlet temperature of the core (outlet temperature of steam generator) $T_{i}$, is obtained from Eq. (20) by setting $z=L_{s} / 2$;

$$
T_{i}=T_{s e c}+\left(T_{o}-T_{s e c}\right) e^{-\frac{\bar{U}_{1} \pi D_{s} n}{C W} \frac{L_{S}}{2}}
$$

Invoking the Boussinesq approximation in the solution of Eq. (1), gives the expected result that the mean velocity, $u$, and hence the flow rate, $W$, are uniform around the loop. Since the density is assumed constant, Eq. (12) takes the form;

$$
W=\frac{\widehat{W}}{\bar{\rho}_{o}} \sqrt{\rho_{l}\left(\bar{\rho}_{2}-\bar{\rho}_{1}\right) \frac{\overline{f_{o}}}{\bar{f}}}
$$

The value of $\bar{\rho}_{1}$ is obtained from volume averaging of densities over the up-flow side of the PWR system elements, as follows:

$$
\bar{\rho}_{1}=\frac{\bar{\rho}_{c} V_{c}+\bar{\rho}_{u} V_{u}+\bar{\rho}_{s} \frac{V_{s}}{2}}{V_{1}}
$$

where $\bar{\rho}_{c}, \bar{\rho}_{u}, \bar{\rho}_{s}$ are the average densities of the core, sum of the hot leg and upper plenum, and steam generator respectively. $V_{c}, V_{u}, V_{s}$, and $V_{1}$ are the volumes of the core, sum of the hot leg and upper plenum, and steam generator respectively and $V_{1}=V_{c}+V_{u}+\frac{V_{s}}{2}$. The average density of each element can be obtained by using Eq. (13) and the temperature distribution of that element.

$$
\begin{gathered}
\bar{\rho}_{c}=\frac{\int_{0}^{L_{c}} \rho_{c} d z}{L_{c}}=\frac{\int_{0}^{L_{c}}\left(a+b T_{c}\right) d z}{L_{c}}=a+b\left(\frac{T_{i}+T_{o}}{2}\right) \\
\bar{\rho}_{u}=\rho_{u}=a+b T_{o}
\end{gathered}
$$




$$
\begin{gathered}
\bar{\rho}_{s}=\frac{\int_{0}^{\frac{L_{s}}{2}} \rho_{s} d z}{\frac{L_{s}}{2}}=\frac{\int_{0}^{\frac{L_{s}}{2}}\left(a+b T_{s)} d z\right.}{\frac{L_{s}}{2}}=a+b T_{s e c}-\frac{b\left(T_{0}-T_{s e c}\right) C W}{\frac{L_{s}}{2} \bar{U}_{1} \pi D_{s} n}\left\{e^{-\frac{\bar{U}_{1} \pi D_{s} n}{C W} \frac{L_{s}}{2}}-1\right\} \\
\bar{\rho}_{2}=\rho_{2}=a+b T_{i}=a+b\left[T_{s e c}+\left(T_{o}-T_{s e c}\right) e^{-\frac{\bar{U}_{1} \pi D_{s} n}{C W} \frac{L_{s}}{2}}\right]
\end{gathered}
$$

Substitute Eqs. (24) through (27) into Eq. (23) to get $\bar{\rho}_{1}$, and then Eq. (22) can be used to evaluate the mass flow rate, $W$

\subsection{Overall heat transfer coefficient}

The overall heat transfer coefficient for the radial flow of heat across a circular tube based on the internal tube surface, $U_{i}$, is [9];

$$
\frac{1}{U_{i}}=\frac{1}{H_{i}}+\frac{A_{i} t_{w}}{A_{m w} K_{w}}+\frac{A_{i}}{A_{o} H_{o}}
$$

where $H_{i}$ and $H_{o}$ are the heat transfer coefficient inside and outside the tube respectively, $A_{i}$ and $A_{o}$ are the internal and external tube surface area respectively, $A_{m w}$ is the mean wall area for heat transfer, and $K_{w}$ is the thermal conductivity of the wall tube. The heat transfer resistance by conduction can be neglected because of small thickness of steam generator tubes. For a single flow in the steam generator, convective heat transfer on the inside of the tubes and nucleate boiling on the secondary side of the tubes will occur [10]. The heat transfer coefficients in the primary side of steam generator $H_{i}$, have been taken, from forced flow correlations for single phase as [8]:

$$
H_{i}=0.023 \operatorname{Re}^{0.8} \operatorname{Pr}^{0.3} \frac{k}{d_{e}}
$$

where Re and Pr are the Reynolds and Prandtle numbers respectively, $k$ is the fluid conductivity, and $d_{e}$ is the equivalent diameter of the flow channel. The heat transfer coefficient for nucleate boiling of the secondary side of steam generator $H_{o}$, can be taken from the following correlation [8]:

$$
H_{o}=2.5551 \times 10^{-3} e^{0.6446133 P_{s e c}}\left(T_{b u l k}-T_{\text {sec }}\right)^{3}
$$

where $P_{\text {sec }}$ is the secondary side pressure in $\mathrm{MPa}, T$ in ${ }^{\circ} \mathrm{C}$ and $H_{\mathrm{o}}$ in $\mathrm{kW} / \mathrm{m}^{2} .{ }^{\circ} \mathrm{C}$.

\section{Results}

It is important to note that the values of NC parameters are calculated iteratively. At a certain power $Q_{o}$, the values of $\rho_{o}$ and $f_{o}$ are calculated when the inventory is $100 \%$ and under a given initial primary pressure $\left(P_{o}=11.2 \mathrm{MPa}\right)$. The values of $\bar{\rho}_{o}$ and $\bar{f}_{o}$ for each core power, $Q_{o}$, are listed in Table 1.

Table 1: Values of $\bar{\rho}_{o}$ and $\bar{f}_{o}$ as a function of core power.

\begin{tabular}{|c|c|c|c|}
\hline $\boldsymbol{Q}_{\boldsymbol{o}}(\mathbf{K W})$ & \multicolumn{1}{|c|}{30} & 60 & 100 \\
\hline$\overline{\boldsymbol{\rho}}_{\boldsymbol{o}}$ & 743.2258 & 734.6119 & 730.6987 \\
\hline$\overline{\boldsymbol{f}}_{\boldsymbol{o}}$ & $4.847 \times 10^{-3}$ & $4.614 \times 10^{-3}$ & $4.462 \times 10^{-3}$ \\
\hline
\end{tabular}


The predictions of the PWR model described previously are compared with experimental data from the Semiscale 2A system [10]. The experiments were conducted at three different steady-state powers (30, 60 and $100 \mathrm{KW})$. Table 2 summarizes the relevant data concerning the Semiscale Mod-2A system [10, 11, 12]. The behaviours of the NC as a function of primary pressure and the comparisons between the experimental and theoretical results are shown in 3-6.

Table 2 Summary of the design and operating data for the Semiscale model.

\begin{tabular}{|c|c|c|c|c|c|}
\hline Parameter & Data & Parameter & Data & Parameter & Data \\
\hline$A_{i}$ & $3.05 \times 10^{-4} \mathrm{~m}^{2}$ & $L_{\mathrm{s}}$ & $20 \mathrm{~m}$ & $P_{\mathrm{sec}}$ & $6 \mathrm{MPa}$ \\
\hline$A_{o}$ & $3.87 \times 10^{-4} \mathrm{~m}^{2}$ & $\bar{\nabla}_{o}$ & $0.165 \mathrm{~m}^{3}$ & $T_{\mathrm{sec}}$ & $275.64{ }^{\circ} \mathrm{C}$ \\
\hline$D_{\mathrm{s}}$ & $0.0222 \mathrm{~m}$ & $V_{\mathrm{c}}$ & 0.06 & $T_{1}$ & $T_{\text {bulk }}$ \\
\hline$t_{w}$ & $0.00124 \mathrm{~m}$ & $V_{\mathrm{u}}$ & 0.18 & $\rho_{1}$ & $\rho_{\text {bulk }}$ \\
\hline$n$ & 6 & $V_{\mathrm{s}}$ & 0.22 & $\widehat{W}$ & $1.35 \mathrm{Kg} / \mathrm{Sec}$ \\
\hline$L_{\mathrm{c}}$ & $3.66 \mathrm{~m}$ & $V_{\mathrm{cl}}$ & 0.29 & $\begin{array}{c}\text { full core power } \\
\text { (scaled) }\end{array}$ & $2 \mathrm{MW}$ \\
\hline
\end{tabular}

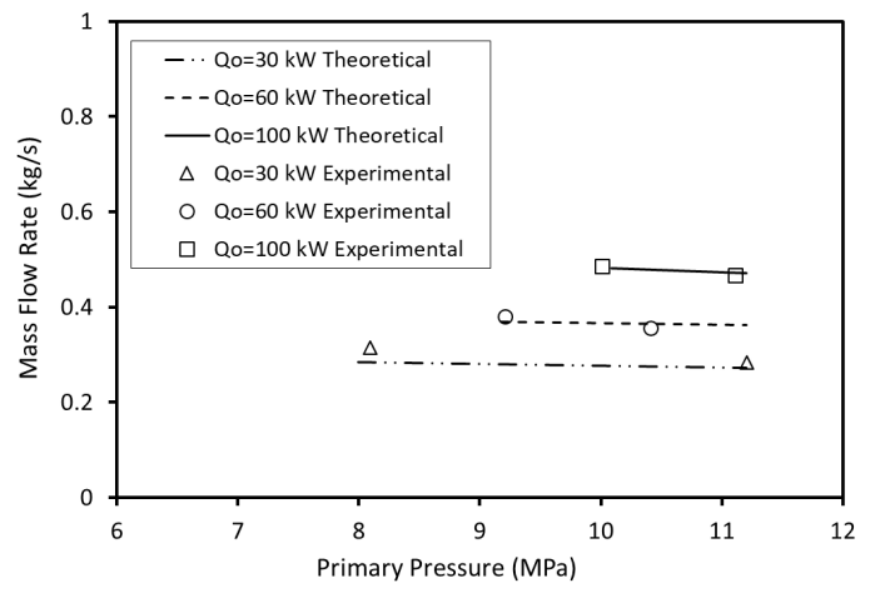

Fig. 3 Comparison of theory with Semiscale PWR data of Mass flow rate versus Primary pressure for different core powers. 


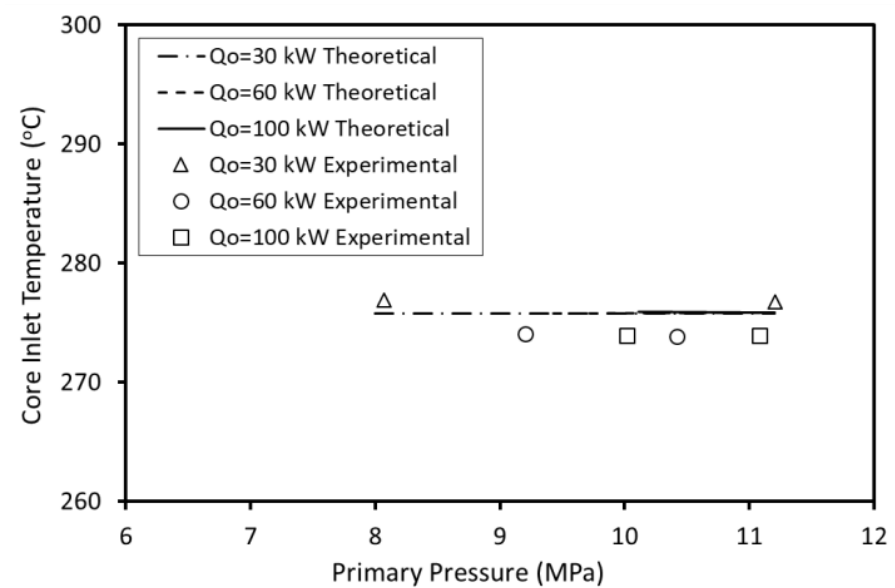

Fig. 4 Comparison of theory with Semiscale PWR data of Core inlet temperature versus Primary pressure for different core powers.

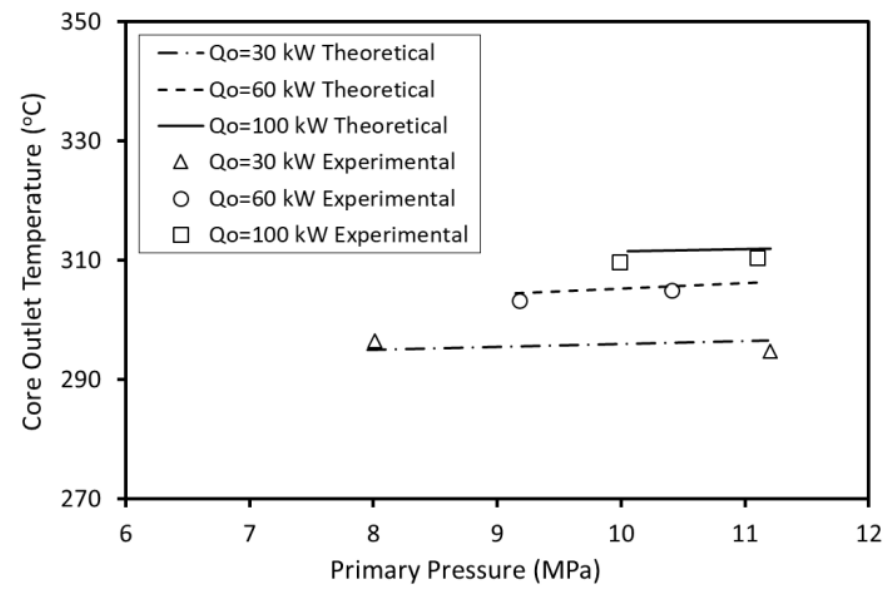

Fig. 5 Comparison of theory with Semiscale PWR data of Core outlet temperature versus Primary pressure for different core powers.

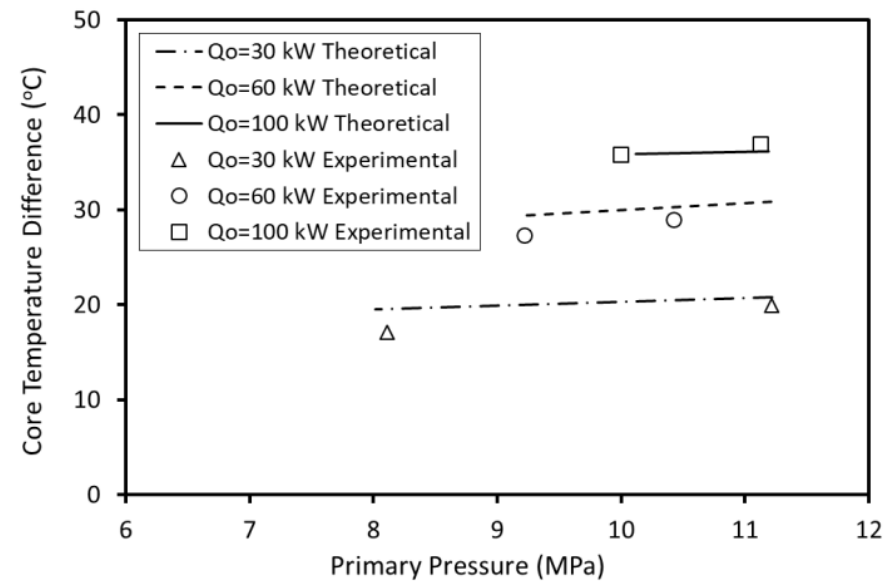

Fig. 6 Comparison of theory with Semiscale PWR data of Core temperature difference versus Primary pressure for different core powers. 
For single-loop system, the mass flow rate in Fig. 3 is found to vary considerably, depending on system pressure. Starting at blow down initiation $(P=11.2 \mathrm{MPa})$, the mass flow rate is seen to increase very slowly as the primary pressure is decreased and, thus to be relatively insensitive to it. This is due to the fact that single-phase water is essentially non compressible and hence the overall gravity head (which is the driving potential) is relatively unaffected by changes in pressure.

Figure 4 shows the core inlet temperature as a function of primary pressure. Again, it is observed that the cold leg temperature is unaffected by changes in primary pressure because, the heat transfer surface area in the up-flow side of the steam generator is sufficient to allow the primary fluid temperature to equilibrate with the secondary fluid temperature within the up-flow side of the steam generator. As the hotter sub-cooled fluid entering the steam generator from the core travels upward, convective heat transfer on the inside of the tubes and nucleate boiling on the secondary side will cause the primary temperature to equilibrate with the secondary temperature.

Figures 5 and 6 show the core outlet temperature (hot leg temperature) and core temperature difference as a function of primary pressure respectively. The hot leg temperature and core temperature difference are relatively insensitive to primary pressure since it was observed earlier that both the mass flow rate and cold leg temperature likewise are insensitive to pressure.

Figures 3 to 6 also show the effect of the reactor power on the NC parameters. In general, it can be seen that the overall trends for the three power levels are similar. Also, the increases in core power leads to smaller band of singlephase NC. The primary pressure, at the starting and ending of single-phase NC, is greater the higher the core power. In Fig.3, the loop mass flow rate is observed to increase with increasing core power. This behaviour can be explained as due to the increase in the overall density gradient between the hot and cold sides of the systems, resulting in higher loop mass flow rates. Fig. 4 reveals that the cold leg temperature is practically independent of core power. Figs. 5 and 6 indicate that the hot leg temperature and the core temperature difference tend to increase with increasing core power.

It is noted that the present theory is capable in predicting the correct trend over the whole range of primary pressure for single-phase flow NC and show reasonable agreement with the experimental results. Table 3 shows the maximum error between the experimental and theoretical results of NC parameters. The discrepancies in the results can be attributed to different reasons. The first one is the uncertainties in experimental data, which were estimated [11] as \pm $0.033 \mathrm{Kg} / \mathrm{s}$ for mass flow rate, $\pm 5^{\circ} \mathrm{C}$ for core temperature difference, and $\pm 0.4 \mathrm{MPa}$ for secondary pressure. The second reason is the model approximations. The one-dimensional model used is inadequate, under certain operating conditions and in regions where abrupt changes in flow area occurs, to describe the three-dimensional nature of the flow. Another reason is the usage of forced flow correlations for heat transfer coefficient and friction factor in NC flow and neglecting the effect of form losses in the various components in the loop.

Table 3 Maximum absolute errors between experimental and theoretical results for the NC parameters versus the primary pressure for different power values.

\begin{tabular}{|c|c|c|c|c|}
\cline { 2 - 5 } \multicolumn{1}{c|}{} & \multicolumn{4}{c|}{ Max. error (\%) } \\
\hline Power (KW) & $\boldsymbol{W}$ & $\boldsymbol{T}_{\boldsymbol{i}}$ & $\boldsymbol{T}_{\boldsymbol{o}}$ & $\Delta \boldsymbol{T}_{\text {core }}$ \\
\hline 30 & 10.05 & 0.38 & 0.63 & 14.43 \\
\hline 60 & 2.9 & 0.72 & 1.47 & 7.9 \\
\hline 100 & 1.1 & 0.74 & 6.36 & 2.14 \\
\hline
\end{tabular}

\section{Conclusion}

In this paper, an analytical one-dimensional model has been developed, based on solutions to the continuity, momentum and energy equations and expressions for the natural circulation parameters have been derived for PWR plant. By comparing the theoretical results with the experimental, it has been demonstrated that the simple analytical model is capable of describing the quasi steady-state behaviour during a small break LOCA or loss of pumped flow 
transient. Also, this work demonstrated that natural circulation in a PWR type system can provide an effective mechanism for the rejection of core decay heat to the secondary side of a steam generator.

\section{References}

[1] L. J. Ybarrondo, C. W. Solbrig, and H. S. Isbin, "The Calculated loss of coolant accident (a review)," American Institute of Chemical Engineers, Monograph series No. 7, vol. 68, 1972.

[2] E. E. Lewis, Nuclear power reactor safety. Wiley, New York, 1977.

[3] Y. Zvirin, P. R. III Jeuck, C. W. Sullivan, and R. B. Duffey, "Experimental and analytical investigation of a natural circulation system with parallel loops," ASME Journal of Heat Transfer, vol. 103, pp. 645-652, 1981.

[4] M. W. Abdulrahman, "Heat transfer in a tubular reforming catalyst bed: Analytical modelling," in proceedings of the 6th International Conference of Fluid Flow, Heat and Mass Transfer (FFHMT'19), 2019.

[5] M. W. Abdulrahman, "Exact analytical solution for two-dimensional heat transfer equation through a packed bed reactor," in Proceedings of the $7^{\text {th }}$ World Congress on Mechanical, Chemical, and Material Engineering (MCM'20), 2020.

[6] W. E. Burchill, and P. E. Abramson, PWR small break LOCA. Guidebook to light water reactor safety analysis, Chap. 3, P. B. Abramson, ed., Hemisphere, New York, 1985.

[7] J. B. Keller, "Oscillations in a model of thermal convection," J. Fluid Mech., vol. 26, pp. 599-606, 1966.

[8] A. Shitzer, D. Kalmanoviz, Y. Zvirin and G. Grossman, "Experiments with a flat-plate solar water heating system in thermosyphonic flow," Solar Energy, vol. 22, pp. 27-35, 1979.

[9] M. M. El-Wakil, Nuclear power engineering. Mc Graw-Hill Book Company, 1962.

[10] G. G. Loomis and K. Soda, "Results of the Semiscale Mod-2A natural circulation experiments," NRC Report, Idaho National Engineering Lab., NUREG / CR-2335, September, 1982.

[11]D. J. Shimeck and G. W. Johnsen, "Natural circulation cooling in a pressurized water reactor geometry aunder accident-induced conditions," Nucl. Sci. Eng., vol. 88, pp. 311-320, 1984.

[12] R. B. Duffey and J. P. Sursock, "Natural circulation phenomena to small breaks and transients," Nuclear Engineering and Design, vol. 102, pp. 115-128, 1987. 